\title{
Interplay of spin density wave and superconductivity with different pairing symmetry
}

\author{
Haranath Ghosh \\ Department of Physics, University of Arizona, Tucson, AZ 85721, USA. \\ S. Sil \\ Theoretische Tieftemperaturephysik, Gerhard Mercator Universität, Duisburg 470 48, Germany. \\ S. N. Behera \\ Institute of Physics, Sachivalaya Marg, Bhubaneswar -751 005, INDIA.
}

\begin{abstract}
A model study for the coexistence of the spin density wave and superconductivity is presented. With reference to the recent angle resolved photo emmission experimental data in high $T_{c}$ cuprates, presence of the nested pieces of bands is assumed. The single band Hubbard model, therefore, when treated within the Hatree-Fock mean field theory leads to a spin density wave (SDW) ground state. The superconductivity (SC) is assumed to be due to a generalised attractive potential with a separable form without specifying to any particular origin. It therefore allows a comparative study of the coexistence of superconductivity of different order parameter symmetry with the spin density wave state. We find that the phase diagram, comprising of the amplitudes of the respective gaps (SC and SDW) Vs. band filling resembles to that of the high $T_{c}$ cuprates only when the order parameter of the superconducting phase has $d$-wave symmetry. Thermal variation of different order parameters (e.g, SC and SDW) also show interesting coexistence and reentrance behaviors that are consistent with experimental observations, specially for the borocarbides.
\end{abstract}

75.30.F, 74.70. Hk, 75.10.J, 74.20.F

\section{INTRODUCTION}

The coexistence of magnetism and superconductivity has intrigued both experimentalists and theoreticians for a long time. It is well known that the superconductivity (SC) and spin density wave (SDW) co-exist in a large classes of systems e.g., organic layered superconductors [1], the Bechgaard salts $(T M T S F) X$, with $X=P F_{6}$, $\mathrm{AsF}_{6}, \mathrm{ClO}_{4}$ etc. [2], heavy fermion systems [3], high temperature superconductors [4] and recently discovered borocarbides [5].

In case of the organic superconductors belonging to the family $(T M T S F)_{2} X \quad(T M T S F \equiv$ tetramethyltetraselena fulvalence and $X=P F_{6}, A s F_{6}$ etc.) show coexistence of superconductivity and spin density wave like antiferromagnetism (SDW) at low temperature in moderate pressures $(\sim 7 \mathrm{Kbar})\left[6\right.$. Many compounds $\left(X=P F_{6}\right.$, $A s F_{6}, S b F_{6}$ etc.) exhibit metal - insulator transition at $12-17 \mathrm{~K}$ under ambient pressure which is caused by spin density wave (SDW) transition. This transition is suppressed by moderate pressures that results in superconductivity. However, the nature of this coexistence is very much different from what would encounter in case of ternary compounds. In organic superconductors, there is only one conduction band and the antiferromagnetic ordering destroys the Fermi surface (FS) due to its $2 k_{F}$ periodicity which results in an insulating phase. In high$T_{c}$ cuprates also due to the low dimensionality aspects there exists similar periodicity due to nesting of the FS [7], and leads to FS instability by forming the SDW state. On the other hand, in intermetallic compounds the mag- netism is due to localized $f$ electrons and superconductivity arises due to the conduction electrons. The organic superconductors in turn, are essentially quasi one dimensional compounds with weak interaction between the adjacent chains. Our main concern in the present paper will however be to discuss the coexistence of antiferromagnetism (SDW) and superconductivity (SC) in the cuprates.

In the high temperature cuprate superconductors $\mathrm{La}_{2-\mathrm{x}}(\mathrm{Sr}, \mathrm{Ba})_{\mathrm{x}} \mathrm{CuO}_{4-\mathrm{y}}$ and $\mathrm{YBa}_{2} \mathrm{Cu}_{3} \mathrm{O}_{7-\mathrm{y}}$ strong antiferromagnetic spin order with a commensurate wave vector $\mathrm{Q}=(\pi / \mathrm{a}, \pi / \mathrm{b})$ has been observed in neutron [8] and Raman scattering [9] experiments. Moreover, in contrast to the conventional low $T_{c}$ superconductors, the coherence length of high- $T_{c}$ materials is short (10 to $\left.20 \rho A\right)$; the average magnetic field seen by the cooper pairs need not be zero. Thus it appears that the antiferromagnetism and superconductivity are intimately related in these materials, in contrast to the magnetism opposing superconductivity in conventional superconductors.

In the context of the influence of spin correlation on superconductivity in oxide systems there exists two limiting points of view ; $(i)$ localized picture where $U \gg W$ and (ii) itinerant picture $U \leq W$; where $U$ is the strength of the onsite coulomb repulsion and $W$ is the band width. In the large $U$ limit, the valence electrons are localized on each $\mathrm{Cu}$-site. To lowest order in $t / U$ there is an antiferromagnetic exchange interaction $J$ of the order of $t^{2} / U$, which produces spin ordering $(t$ being the hopping integral) 10. In the second point of view, $U$ is assumed to be small so that the Mott Hubbard transition does not takes 
place and Bloch states form a convenient basis to describe the system. Now for a perfect square lattice and at halffilling, the bands get nested with certain nesting wave vector $\vec{Q}$ that determines a new super periodicity leading to spin density wave ground state which may or may not be comensurate with the underlying lattice. The spin density wave ground state causes an energy gap $2 \Delta_{S D W}$ at the Fermi surface of the undoped material, leading to an insulator. Here we shall confine our selves to the latter point of view (i.e. $U \ll W$ ).

The SDW ground state is obtained from the single band Hubbard model within the Hatree-Fock approximation and assuming that the nesting of the FS exists only in certain directions of the FS. The directions of the FS where nesting exists will be instable with respect to the SDW formation whereas the superconducting instability may occur in the rest of the part of the FS, provided there exists some attractive interaction between the quasi-particles mediated by some boson exchange. Now, as regards the high- $T_{c}$ cuprates are concerned the knowledge of the exact mechanism for the high temperature superconductivity is yet to be known. In confirmity with the role of electronic correlations, the parent (undoped) cuprate materials are always antiferromagnetic (SDW state in our case) but the long range AF order vanishes sharply on doping the system. However, there is some evidence that the AF fluctuations exists even in the superconducting phase. Hence there have been proposals among the others that the pairing might as well arise due to the exchange of quanta of AF fluctuations [11. In that case, the superconducting order parameter will have a $d$-wave symmetry. On the other hand, the SC in the system may even be caused by the quanta of the fluctuations of the collective (amplitude and phase) modes of the SDW state, present in our model, as proposed by Schrieffer et al., 112] and others 13] orelse the SC may even be due to normal phonon mediated mechanism. In either of these cases the nature of the superconducting order parameter symmetry could be different depending on the nature of the origin of the pairing mechanism. Furthermore, there are experimental indications that the symmetry of the order parameter of the high- $T_{c}$ superconductors could be an admixture of $s$ and $d$ - wave like (i.e, $s \pm d$ or $\mathrm{d}+$ is) [14]. Therefore, we present a model study for the coexistence of SDW with SC where the SC is caused by some generalised attractive potential with separable form (without specifying any particular mechanism) leading to different order parameter symmetry. It therefore incorporates, two competing physical processes involving the electron-hole (SDW) like pairing of opposite spins with a net momentum difference $(\vec{Q})$ between the conjugates and electron-electron (SC) pairing of opposite spins with zero total momentum. Such a model study can also provide an important understanding regarding the effect of the presence of antiferromagnetic
(AF) order on the pairing symmetry of the superconducting state, which is extremely important for high- $T_{c}$ cuprates as well as the recently discovered borocarbides. Our main concern therefore boils down to study of the phase diagram, comprising of the gap magnitudes of the respective gaps e.g, the SDW and the SC gaps as a function of band filling (hole concentration). Interestingly enough, we obtain the phase diagram which resembles to that of the high- $T_{c}$ superconductors only when the superconducting order parameter has the $d$-wave symmetry.

The rest of the paper is arranged as follows. In section II we provide a brief mean field theory of the SDW ground state from a single band Hubbard model. Section III contains the mean field model Hamiltonian for the coexistence of the SDW and SC together with the derivation of the respective gap equations. Section IV is devoted to discuss the results obtained from our model calculatons and finally we conclude in section V.

\section{SPIN DENSITY WAVE STATE}

The SDW state is a kind of antiferromagnetic state with the electronic spin density forming a static wave. The density varies perpendicularly as a function of position with no net magnetisation in the entire volume. The SDW transition occurs when the spatial spin density modulation is due to delocalization or initinerant electrons rather than the localized one. Usually in the normal state the density $\rho_{\uparrow}(\vec{r})$ of electron spins polarized upward with respect to any quantization axis is completely cancelled by $\rho_{\downarrow}$ of downward polarized spins. In the SDW state, however, the difference $\sigma(\vec{r})=\rho_{\uparrow}(\vec{r})-\rho_{\downarrow}(\vec{r})$ is finite and modulate in space as a function of the position vector $\vec{r}$ in the SDW state. Such tendency of forming SDW ground state takes place when a system possesses nested pieces of FS together with intermidiate coulomb correlation. In the case of the SDW transition it is the wave vector dependent static magnetic susceptibility which develops a singularity at $\vec{q}=\vec{Q}$ i.e., $\left.\chi(q, \omega)\right|_{\vec{q}=\vec{Q}, \omega=0}=<<$ $S^{+}(q, t) ; S^{-}(-q, t)>>\left._{\omega}\right|_{q=Q, \omega=0} \rightarrow \infty$ where $S^{ \pm}$are the spin raising and lowering operators and $\vec{Q}$ is known as the nesting wave vector which determines the periodicity of the SDW. This singularity in the magnetic susecptibility is an artifact of the nesting property of the FS given by

$$
\epsilon_{\vec{k}}=-\epsilon_{\vec{k}+\vec{Q}}
$$

In addressing the possibilty of the coexistence of SDW and $\mathrm{SC}$ ordering in $\mathrm{CuO}$ based high- $T_{c}$ material we will look for a model of a single $\mathrm{CuO}$ layer and neglect the inter-layer hopping because inter-layer hopping is weak compared to the intra-layer hopping integral. The simpliest model one can use to describe the antiferromagnetic ordering of the two dimensional correlated system is the Hubbard model on a square lattice. 


$$
H=-\sum_{<i j>\sigma} t_{i j} C_{i \sigma}^{\dagger} C_{j \sigma}+U \sum_{i} \hat{n}_{i \uparrow} \hat{n}_{i \downarrow}
$$

where $t_{i j}$ is hopping integral between the different orbitals $i \& j, \hat{n}_{i \sigma}=C_{i \sigma}^{\dagger} C_{j \sigma} \equiv$ number operator and $\mathrm{U}$ is the intratomic coulomb repulsion or the cost in energy to put two electrons on the same site. The interaction term in the Hubbard Hamiltonian may be treated in the mean field approximation to get the SDW state as follows,

$$
\begin{aligned}
H_{I}= & U \sum_{i} n_{i \uparrow} n_{i \downarrow}=U \sum_{i} C_{i \uparrow}^{\dagger} C_{i \uparrow} C_{i \downarrow}^{\dagger} C_{i \downarrow} \\
& \approx U \sum_{i}\left[<C_{i \uparrow}^{\dagger} C_{i \uparrow}>C_{i \downarrow}^{\dagger} C_{i \downarrow}+C_{i \uparrow}^{\dagger} C_{i \uparrow}<C_{i \downarrow}^{\dagger} C_{i \downarrow}>\right. \\
& \left.-<C_{i \downarrow}^{\dagger} C_{i \uparrow}>C_{i \uparrow}^{\dagger} C_{i \downarrow}-<C_{i \uparrow}^{\dagger} C_{i \downarrow}>C_{i \downarrow}^{\dagger} C_{i \uparrow}\right] \\
= & \sum_{i}\left[U n_{i}\left(C_{i \uparrow}^{\dagger} C_{i \uparrow}+C_{i \downarrow}^{\dagger} C_{i \downarrow}\right)+\Delta_{i}^{z}\left(C_{i \uparrow}^{\dagger} C_{i \uparrow}-C_{i \downarrow}^{\dagger} C_{i \downarrow}\right)\right. \\
& \left.+\Delta_{i}^{+} C_{i \downarrow}^{\dagger} C_{i \uparrow}+\Delta_{i}^{-} C_{i \uparrow}^{\dagger} C_{i \downarrow}\right]
\end{aligned}
$$

where $n_{i}=\frac{1}{2}\left(<C_{i \uparrow}^{\dagger} C_{i \uparrow}>+<C_{i \downarrow}^{\dagger} C_{i \downarrow}>\right)$, $\Delta_{i}^{z}=-\frac{U}{2}\left(<C_{i \uparrow}^{\dagger} C_{i \uparrow}>-<C_{i \downarrow}^{\dagger} C_{i \downarrow}>\right)=-U<S_{z}>$, $\Delta_{i}^{+}=-U<C_{i \uparrow}^{\dagger} C_{i \downarrow}>=-U<S_{i}^{-}>$and $\Delta_{i}^{-}=-U<C_{i \downarrow}^{\dagger} C_{i \uparrow}>=-U<S_{i}^{-}>$

Therefore, the meanfield Hubbard Hamiltonian can be written as,

$$
H=-\sum_{i j \sigma} t_{i j} C_{i \sigma}^{\dagger} C_{j \sigma}+U \sum_{i} n_{i} \hat{n}_{i}+\sum_{i} \bar{\sigma}_{i} \cdot \bar{B}_{i}
$$

where $\hat{n}_{i}=C_{i \uparrow}^{\dagger} C_{i \uparrow}+C_{i \downarrow}^{\dagger} C_{i \downarrow}$ and $B_{i}^{z}=\Delta_{i}^{z}=-U<$ $S_{i}^{z}>\equiv$ the order parameter for longitudinal magnetization, $\left.B_{i}^{ \pm}=\Delta_{i}^{ \pm}=-U<S_{i}^{ \pm}\right\rangle \equiv$ transverse magnetization and $\bar{\sigma}_{i}=\left(C_{i \uparrow}^{\dagger} C_{i \downarrow}^{\dagger}\right) \bar{\tau}\left(\begin{array}{c}C_{i \uparrow} \\ C_{i \downarrow}\end{array}\right)$, where $\bar{\tau} \equiv$ Pauli matrices. The $2^{\text {nd }}$ term of equation (4) corresponds to total charge of the system whereas the $3^{r d}$ term is the total spin. Hence, eqn. (4) demonstrates that the charge and spin degree of freedom of the Hubbard model is seperated. Now, the SDW state can be described equivalently in terms of either the longitudinal or transverse spin polarization. For example the SDW state with wave vector $\vec{Q}$, having longitudinal spin polarization is

$$
<S_{i}^{z}>=S_{o}^{z} \times\left\{\begin{array}{c}
\cos \left(\vec{Q} \cdot \vec{R}_{i}\right) \text { for } \mathrm{SD} \text { on sites. } \\
\sin \left(\vec{Q} \cdot \vec{R}_{i}\right) \text { for } \mathrm{SD} \text { on bonds. }
\end{array}\right.
$$

In momentum representation the mean field Hamiltonian for a transverse SDW state becomes

$$
\begin{aligned}
H_{S D W}= & \sum_{k, \sigma}\left(\epsilon_{k}-\mu\right) C_{k, \sigma}^{\dagger} C_{k, \sigma}+\Delta_{S D W} \sum_{k}\left(C_{k+Q \uparrow}^{\dagger} C_{k \downarrow}\right. \\
& +h . c)
\end{aligned}
$$

where $\Delta_{S D W}=-U \sum_{k}<C_{k \uparrow}^{\dagger} C_{k-Q \downarrow}>$, the order parameter for the transverse SDW state. The quasi particle energy spectrum of the SDW state can be writen as $E_{k}=\sqrt{\left(\epsilon_{k}-\mu\right)^{2}+\Delta_{S D W}^{2}}$ with a gap of $2 \Delta_{S D W}$ at the Fermi level. To note, there is a formal similarity of the SDW mean field theory with that of the BCS, in energy spectrum, gap equation, as well as in the collective modes (it is $2 \Delta_{S D W}$ in case of the SDW state).

\section{THE COEXISTENCE OF SDW AND SC}

Usually, magnetism and superconductivity are expected to be mutually exclusive phenomena i.e, they are unlikely to occur simultaneously in the same compound. Superconductivity (including in the high temperature superconductors) is known to be due to Cooper pair formation of electrons of opposite spins and momenta whereas magnetism requires spin polarisation. Therefore, naturally one order would inhibit the other. Furthermore, like superconductivity (electron-electron pairing), the transverse SDW state is also a result of condensation of electron-hole pairs of opposite spins but with a momentum difference of $\vec{Q}$ between the conjugates. Hence, when any of the orders (either the SC or the SDW) set in, the FS is instable with respect to that 'condensate state'. In other words, if one of the phases (say SDW) sets in first, and exists all over the FS, then there will be no carrier available to form cooper pairs and hence no superconductivity. This would also be equally true in case of the SDW state, had the superconductivity appeared first. However, in reality we do see the coexistence of the two phases as is already discussed earlier. In what follows we discuss two different scenarios where both the presence of SDW state and SC coexist.

\section{A. The case of an isotropic SDW-state : SC can arise over the SDW backg round}

If the nesting of the FS is perfect the entire FS could be isotropically gapped due to the formation of the SDW state transforming the system from a metal to an insulator. In order to build superconductivity over such an insulating phase one needs to dope the system with charge carriers. In the event of doping the system with holes, or equivalently removal of electrons from the lower filled (valence) SDW band, there will be deviation from perfect nesting of the FS resulting in a local suppression of the SDW gap. This is so because, the gain in electronic energy resulting from the formation of the SDW state is lowered due to removal of electrons from the valence band. This local suppression of the SDW gap acts as a potential well for the injected hole, in which it gets 
self-trapped forming the so called spin bag. On creating two holes it is energetically favourable for them to dig a deeper well and stay together provided the two holes have opposite spins to avoid Pauli exclusion principle. This is however nothing but a local Cooper pair and if such bags with two holes of opposite spins move coherently, the system will be superconducting. This is the essence of Schrieffer's spin bag model for high temperature superconductivity 12 and hence is an example where superconductivitycan arise over the SDW state.

\section{B. Anisotropic SDW state : SC can coexist with SDW}

The above case is however, physically resonable only when the hole concentration is very small. But in reality superconductivity in most of the systems (specially high $T_{c}$ systems) appears only after large doping. So, it is likely that the SDW state will be completely suppressed in particular directions of the FS whereas it would still exist in the rest of the FS which still nests (anisotropic SDW). Such situation may also appear due to particular topology of the FS of a system without doping i.e, the system may have nested pieces of FS only in certain direction and no nesting in other direction resulting in an anisotropic SDW state. In the regions of the FS where the SDW gap vanishes (i.e, the lower and upper SDW bands merge together), the pairing interaction between the SDW quasi particles can take place leading to superconductivity. From a microscopic point of view, the effective attractive interaction between the SDW quasi particle in this picture can be rationalised (Ghosh and Sardar [13]) as arising due to the exchange of the collective modes of the SDW state. In both the cases discussed above, the origin of superconductivity is fundamentally different from that in conventional superconductors.

In contrast, in the cases where nesting is not perfect either due to peculiar topology of the FS or due to doping, the SDW gap will appear only in the nested part of the FS allowing for the superconducting instability in the rest of the FS. But in such a case, the origin of superconducting pairing may arise be due to any other mechanism including the BCS phonon exchange 15]. Besides, at least in the case of high- $T_{c}$ SCs there are other proposals for SC like the charge transfer mechanism [16] and the pair tunneling mechanism [17] etc. However, the nature of SC pairing and the symmetry of the order parameter in the cuprates is still unknown. It was proved by Dzyaloshinskii and Yakovenko [18] from a generalised interaction through renormalisation group equations that the two dimensional Hubbard model could lead to various instabilities including single superconducting $d_{x^{2}-y^{2}}$ pairing, along with possible coherent mixture of charge density wave (CDW) and SDW phases. Ruvalds and coworks 19 also found that $d_{x^{2}-y^{2}}$ pa iring was favoured for nested fermi surfaces consisting of parallel orbit segments.

The Hamiltonian for the coexistence phase of superconductivity and the SDW state can therefore be obtained by adding a pairing interaction term to equation (6) which in the mean field BCS approximation can be written as

$$
\begin{aligned}
H= & \sum_{k, \sigma}\left(\epsilon_{k}-\mu\right) C_{k \sigma}^{\dagger} C_{k \sigma}+\Delta_{S D W} \sum_{k}\left(C_{k+Q \uparrow}^{\dagger} C_{k \downarrow}+\text { h.c. }\right) \\
& +\sum_{k} \Delta_{s c}(k)\left(C_{k \uparrow}^{\dagger} C_{-k \downarrow}^{\dagger}+\text { h.c. }\right)
\end{aligned}
$$

where $\Delta_{s c}(k)=-\sum_{k, k^{\prime}} V_{k, k^{\prime}}<C_{k^{\prime} \uparrow}^{\dagger} C_{-k^{\prime} \downarrow}>, V_{k, k^{\prime}}$ being the strength of the attractive pairing interaction mediated by some boson exchange. Depending on the nature of pairing interaction the superconducting gap function exhibits different types of symmetry structure.

In a weak coupling pairing theory, the SC gap parameter satisfies the equation,

$$
\Delta_{s c}(k)=\sum_{k^{\prime}} V\left(k, k^{\prime}\right) \frac{\Delta_{s c}\left(k^{\prime}\right)}{2 E_{k^{\prime}}} \tanh \frac{\beta E_{k^{\prime}}}{2}
$$

where $E_{k}=\sqrt{\epsilon_{k}^{2}+\Delta_{s c}^{2}(k)}$ is the energy of the superconducting quasiparticles. While $\Delta_{s c}(k)$ is not gauge invariant and therefore cannot be directly observed, $\left|\Delta_{s c}(k)\right|^{2}$ is observable. For a nodeless $\Delta_{k}\left(\equiv \Delta_{s c}(k)\right)$, the state is non-degenarate and $\left|\Delta_{k}\right|^{2}$ has the symmetry of the crystal. However, if $\Delta_{k}$ has nodes in certain directions of $\mathrm{k}$, there may be several degenerate pairing states and $\left|\Delta_{k}\right|^{2}$ need not exhibit the point symmetry of the crystal - which is usually referred to as unconvensional pairing state. More precisely, the symmetry of the order parameter is determined by the form of the potential $V$ and the band structure. In the simpliest assumption the band energies are independent of the direction in the $a-b$ plane and hence the interaction potential can be approximated as a separable one, i.e,

$$
V_{k, k^{\prime}}=-V \eta_{k} \eta_{k^{\prime}}
$$

where (a) $\eta_{k}=$ constant, corresponds to an isotropic s-wave (BCS) symmetry ; (b) $\eta_{k}=\mathrm{f}(\mathrm{k})$, refers to an anisotropic s-wave symmetry, provided $\mathrm{f}(\mathrm{k})$ is a smooth function in the first Brillouin zone (BZ) and is +ve definite (i.e, nodeless) (c) $\eta_{k}=\cos \left(k_{x} a\right)+\cos \left(k_{y} a\right)$, correponds to an extended s-wave $\left(s^{\star}\right)$ pairing symmetry whereas (d) $\eta_{k}=\cos \left(k_{x} a\right)-\cos \left(k_{y} a\right)$ corresponds to $d_{x^{2}-y^{2}}$ pairing symmetry which also changes sign (like (c)) but does not transform as the identity under the full crystal group.

Now, in order to study the interplay between the SDW and the superconducting state (with different possible pairing symmetry as discussed above) we need to calculate the self-consistent gap equations for the respective 
gaps (e.g, SDW \& SC). For this purpose it is convenient to write the Hamiltonian (7) in diagonalised form using four component Nambu operator [21] as follows,

$$
H_{\text {diag }}=\sum_{k} \psi_{k}^{\dagger}\left(\epsilon_{k} \rho_{3} \sigma_{3}+\Delta_{s c} \rho_{3} \sigma_{1} \Delta_{S D W} \rho_{1} \sigma_{3}\right) \psi_{k}
$$

where the four component Nambu operators are defined as,

$$
\psi_{k}^{\dagger}=\left(\begin{array}{llll}
c_{k \uparrow}^{\dagger} & c_{-k \downarrow} & c_{k+Q \downarrow}^{\dagger} & c_{-k-Q \uparrow}
\end{array}\right)
$$

and $\sigma, \rho$ are $4 \times 4$ matrices defined as,

$$
\sigma_{i}=\left(\begin{array}{cc}
\tau_{i} & 0 \\
0 & \tau_{i}
\end{array}\right)
$$

$\tau_{i}$ being the $2 \times 2$ Pauli matrices and $\rho_{i}$ are given by

$$
\begin{aligned}
\rho_{1} & =\left(\begin{array}{llll}
0 & 0 & 1 & 0 \\
0 & 0 & 0 & 1 \\
1 & 0 & 0 & 0 \\
0 & 1 & 0 & 0
\end{array}\right), \rho_{2}=\left(\begin{array}{cccc}
0 & 0 & -i & 0 \\
0 & 0 & 0 & -i \\
i & 0 & 0 & 0 \\
0 & i & 0 & 0
\end{array}\right) \\
\rho_{3} & =\left(\begin{array}{cccc}
1 & 0 & 0 & 0 \\
0 & 1 & 0 & 0 \\
0 & 0 & -1 & 0 \\
0 & 0 & 0 & -1
\end{array}\right)
\end{aligned}
$$

The gap equations corresponding to the order parameters $\Delta_{S D W}$ and $\Delta_{s c}$ defined in equations $(6,7)$ can be calculated by using equation (10) which are given below along with the equation for the total number of charge carriers.

$$
\Delta_{S D W}=\left(-\frac{U}{4}\right) \sum_{k, i=1,2}(-1)^{i} \frac{\Delta_{i}(k)}{E_{i}(k)} \tanh \frac{\beta E_{i}(k)}{2}
$$

and

$$
\begin{gathered}
\Delta_{s c}(k)=\left(\frac{1}{4}\right) \sum_{k^{\prime} i=1,2} V_{k, k^{\prime}} \frac{\Delta_{i}\left(k^{\prime}\right)}{E_{i}\left(k^{\prime}\right)} \tanh \frac{\beta E_{i}\left(k^{\prime}\right)}{2} \\
n=1-\frac{1}{2} \sum_{k, i=1,2} \frac{\left(\epsilon_{k}-\mu\right)}{E_{i}(k)} \tanh \frac{\beta E_{i}(k)}{2}
\end{gathered}
$$

with

$$
\begin{aligned}
E_{i}(k)= & \sqrt{\left(\epsilon_{k}-\mu\right)^{2}+\Delta_{i}^{2}(k)} \text { and } \\
& \Delta_{i}(k)=\left(\Delta_{s c}(k)-(-1)^{i} \Delta_{S D W}\right) .
\end{aligned}
$$

The equations (14-16) are coupled integral equations in the sense that $\Delta_{s c} \equiv \Delta_{s c}\left(\Delta_{s c}, \Delta_{S D W}, n\right), \Delta_{S D W} \equiv$ $\Delta_{S D W}\left(\Delta_{s c}, \Delta_{S D W}, n\right)$ and so is $n \equiv n\left(\Delta_{s c}, \Delta_{S D W}, n\right)$. The self-consistent solutions of these three equations are obtained numerically. Before the numerical results are presented it is worth pointing out that it appears from equations (14-17) as if there exists two effective order parameters $\Delta_{1,2}=\Delta_{s c} \pm \Delta_{S D W}$ indicating that these can interfere with each other either destructively or constructively. In striking contrast, in the coexistent phase of the charge density wave (CDW) and superconductivity [22] no show such interference is obtained where the quasi particle energy spectrum has an effective gap $\sqrt{\Delta_{C D W}^{2}+\Delta^{2}}, \Delta_{C D W}$ being the CDW order parameter. Such a difference in behaviour between the SDW and CDW coexistent with SC may be attributed to the effect of interference between the order parameters of the SDW and SC states both of which involve the pairing of up and down spin quasi-particles, i.e, the electron-hole and electron-electron pairings respectively. Therefore, the electrons with the same spins are likely to compete for both the processes, thereby giving rise to interference.

\section{RESULTS AND DISCUSSION}

The interplay between the SC and SDW state is mainly studied by selfconsistently solving the gap equations (14 - 16) with fixed set of parameters $U=1, V=1.5$, the cut-off energy for SC $\Omega=0.8$, all are in units of the hopping intgral $t$. Two distinct set of results are obtained, (i) for a fixed temperature $(5 \mathrm{~K})$ the amplitudes of the gap functions (SDW \& SC) are obtained for different pairing symmetry as a function of band filling $(n)$ and (ii) temperature variation of the respective order parameters (for different pairing symmetry) are obtained for different (fixed) band fillings. In the former case, the thermal variation of the chemaical potential, $\mu(T)$ is not important whereas for the later the thermal variation of $\mu(T)$ is taken care such that given value of $n$ differs from that of the calculated ones at best at the fifth decimal point. Furthermore, in computing the self-consistent solutions of equations (14 - 16) for different pairing symmetries of the superconducting state, specific forms of the pairing potential and the corresponding order parameters are also assumed. Following the form of the potential in equation (15) we assume the form of the order parameters, for example, for the extended $s\left(s^{\star}\right)$ and $d$ wave SC as, $\Delta^{s(d)}=\Delta^{0}\left(\cos k_{x} \pm \cos k_{y}\right)$.

Now we will discuss the results of our calculation. In fig. 1. we have plotted the isotopic s-wave SDW gap parameter $\left(\Delta_{S D W}\right)$ and isotopic s-wave SC gap parameter $\left(\Delta_{S C}\right)$ evaluated at $\mathrm{T}=5 \mathrm{~K}$ vs. hole concentration $x=1-n$. Clearly the figure demonstrates the strong competition between the two orderings namely SDW and SC emphasizing the coexistence of SDW state and Superconductivity at low hole concentration $x<0.23$. As we increase the hole concentration first SC gap and then the SDW gap goes to zero. As a result $0.23<x<0.25$ we observe a pure SDW phase. When the hole concentration is further increased we obtained pure superconducting phase for $0.25<x<0.5$. This reentrance of superconducting phase with respect to hole concentration is quite 
a new phenomena and to the best of our knowledge no such theoretical demonstartion exists.

In fig.2. we have shown the variation of SDW gap function and amplitude of extended s-wave $\left(s^{\star}\right)$ SC gap function $\Delta_{S C}^{0}$ evaluated at $5 \mathrm{~K}$ with the hole concentration $x$ for the same set of parameters as in Fig. 1. Here there is no such coexistence of SDW and SC phase. In fact SDW phase is dominant in the low hole concentration region where as $\mathrm{SC}$ phase is observed at large hole concentration. More precisely SDW gap shows a maxima at half filling $(x=0)$, decreases with the increase of $\mathrm{x}$ and ultimately vanishes at around $\mathrm{x}=0.5$ where as extended s-wave SC gap is seen at around $x>0.65$ with a maxima around $x=0.8$. Comparing the figure 1 and 2 we find that superconducting ordering suppresses the SDW ordering and vice versa although for isotopic s-wave pairing one finds the coexistence of SDW and SC phase.

In fig. 3. we have presented the scenario of d-wave superconductivity with the SDW. In the undoped $(x=0)$ state the system is an insulating antiferromagnet with a SDW gap. As the system is doped with holes the antiferromagnetic ordering is suppressed and the system becomes superconducting in the region $0.4 \geq x \geq 0.25$. When $x>0.4$ superconductiv ity disappears and we obtain a reentrant SDW phase. However, in this region of hole concentration $(x \geq 0.4)$ the SDW gap parameter is small. Possibly the quantum fluctuation which has been neglected in this mean field calculation will destroy the SDW ordering.

The SDW gap being isotropic $s$ wave it has always maximum value at the half-filling because the density of states (DOS) is maximum at the Fermi lavel (half-filing) only. Since the Fermi surface is determined by the zero energy contour of $\epsilon_{k}=-2 t\left(\cos k_{x} a+\cos k_{y} a\right)=0$, the factor $\left(\cos k_{x} a+\cos k_{y} a\right)$ is therefore smaller, for a square lattice, close to half-filling (as $n=1$ corresponds to the position of the Fermi Surface) and has larger value away from half-filling. Therefore, the self consistent solutions of the amplitudes of the extended $s(d)$ wave superconducting gap will always be minimum (maximum) at halffilling whereas maximum (minimum) at zero filling. This would qualitatively explain the band filling dependence of the extended $s$ wave and $d$ wave superconducting gaps (as is presented in Figures $1-3$ ). The presence of the SDW order makes the situation complicated (and probably more realistic for the systems like the cuprates). The magnitudes of the SDW gap is maximum at half-filling not only because of the large van Hove DOS but also due to the fact that the nesting of the FS is strong close to half-filling. This results in the appearence of the SDW phase at lower doping concentrations $(x)$ irrespective of the nature of the superconducting pairing (cf figures 1-4). Considering the case of interplay between the SDW and $s$ wave superconductivity we therefore find that both the SDW \& SC states are degenerate at $x=0$. However, the SDW phase is more stable than the SC phase al- though the interaction strength is smaller $(U<V)$ due to strong nesting effect. Contribution to superconducting phase at half-filling is probably from the non-nested parts of the FS. However, with doping the nesting of the FS gets affected and superconducting phase becomes stable suppressing the SDW phase (cf Fig. 1, Fig. 3). In case of extended $s$ wave pairing therefore, no strong interplay is found as expected (cf. Fig.2). In case of $d$ wave pairing (cf. Fig. 3) however, the amplitude of the SDW order parameter is large at $x=0$ compared to that for $s$ wave pairing scenario. A closer comparision of the figures $1 \& 3$ will also reveal that the maximum value of $d$ wave SC gap is approximately three times larger than the maximum value of isotropic $s$ wave SC gap (within the same set of all other parameters). This is because for isotropic $s$ SC wave pairing, both the pairing strengths (SC as well as the SDW) are onsite in nature and will be most effective only at the half-filling due to symmetry reason (as discussed earlier). As a result both the orders will inhibit each other. In contrast to isotropic $s$ wave scenario, for $d$ wave pairing the SC phase is however pushed towards higher doped region (unlike being degenerate at $x=0$ ). This is due to the fact that the more extended d-wave pair wave function is more efficient in avoiding the pair breaking effect of the staggered local field. In the conserving fluctuation exchange approximation (FLEX) [20] which are in good agreement with available monte carlo data at higher temperatures showed that when the two dimensional Hubbard model was doped beyond a critical doping the leading instability changes from the SDW channel to $d_{x^{2}-y^{2}}$ pairing channel. Therefore, our results in the figure 3 from a mean field calculation agrees qualitatively with that of FLEX 20] approximation calculation. Furthermore we observe that the gap function for d-wave superconductors decreases sufficiently rapidly from its maximum value with hole concentration in comparison with isotopic s-wave or extended s-wave superconductivity. Finally, in contrast to s-wave pairing d-wave superconductivity does not show any coexistence of SDW and SC phase.

In figure 4 . we have plotted the SDW gap and an admixture of $s$ and $d$ wave pairing $(\mathrm{s}+\mathrm{d})$ superconductivity (with SC gap having $80 \%$ d-wave contribution and $20 \%$ extended s-wave contribution) vs $x$. Here we find that the superconducting phase appears only within very narrow range of hole (almost does not appear) concentration. Also the highest value of the SC gap amplitude is much smaller than that seen for the pure $s$ or $d$ wave picture. This is probably an indication of the fact that the superconducting state with mixed $s+d$ symmetry of the order parameter is stable only in presence of orthrhombic distortion not in tetragonal phase [23]. We shall present results in details of the interplay of the SDW state with complex $(s+i d)$ SC pairing in our next publications.

Fig. $5 \& 6$ demonstrates the thermal variation of the $s$-wave SC gap and the SDW gap for different hole con- 
centrations. In Fig. 5, (for given set of parameters as earlier) for the half-filled case $(x=0.0)$ the SC gap opens up at higher temperatures $\left(T_{c}=161 \mathrm{~K}\right)$, grows with the lowering in temperature but drops to zero at $100 \mathrm{~K}$ till $75 \mathrm{~K}$. On the other hand, the SDW gap opens up at $100 \mathrm{~K}$ and grows with the lowering in temperature but its growth being arrested at $75 \mathrm{~K}$ (due to the re-entrance of the SC state at the same temperature) on further lowering the temperature. Away from half-filling, at $x=0.2$, the SC $T_{c}$ is reduced drastically (to $\sim 85 \mathrm{~K}$ ) and the SCgap drops to zero below $T_{c}$ at $28 \mathrm{~K}$ and reappears at $21 \mathrm{~K}$. The SDW gap (at $x=0.2$ ) grows like a first order transition at around $28 \mathrm{~K}$ (where SC-gap drops to zero) and grows with lowering in temperature which is again being arrested with the reentrance of SC below 21 K. Therefore, Fig. 5 demonstrates very strong interplay and co-existence between the SDW and the $s$-wave state. To be noted the strong interplay between the two ordered phases that includes their coexistence as well as re-entrance, is maximum at half-filling.

While in Fig. 5 there exists coexistence as well as re-entrance of different parameters, Fig.6 demonstrates another particular aspects of their interplay. The hole concentration here is $x=0.1$ and one finds that for certain range of thermal region, around 48 to $65 \mathrm{~K}$ none of the order parameters are stable. In other words, within this thermal regime both the order parameters precipetously either goes to zero or a finite value alternatively for a very small change in temperature. In the rest of the thermal regime it retains the essential features of the earlier figure (Fig.5) as mentioned earlier. The arrest of the growth of the SDW gap at lower temperature with the re-entrance of the SC gap can be understood as follows. The SDW gap (in the mean field treatment) being a very sensitive function of the density of state available at the FS, the opening up of the SC gap takes away large density of states and hence the SDW gap is suppressed at lower temperatures. On the other hand, the rentrance as well as the thermally fluctuating natures are due to typical interefernce between the two order parameters which are described to some extent analytically by the equation (17). Therefore, the present paper presents as extensive study on the influence of the SDW order on the SC pairing symmetry. We would like to further point out that the figures $1 \& 5$ have resemblence with the spin susceptibility data of borocarbides [24, indicating an interplay of magnetism and superconductivity in these systems.

Acknowledgement One of us (HNG) would like to acknowledge the hospitality of Prof. Dr. Peter Entel, through a grant from SFB166, which made it possible for him to visit the Gerhard-Mercator University at Duisburg, Germany where a part of the present work had been carried out. We would also like to thank him for many useful discussions on the subject. One of us (HNG) thanks the Brazilian agency FAPERJ for providing the financial support (Project No. E-26/150.925/96-BOLSA).

[1] T. Ishiguro, K. Yamaji, "Organic superconductors" ; Springer series in Solid state Sciences, Vol. 48, p-99, Berlin, Heidelberg, New York ; Springer 1990.

[2] For a recent review in organic superconductors see, $\mathrm{H}$. Mori, Int. Jr. Mod. Phys. B 8, 1 (1994) and L. N. Bulaevski, Adv. Phys. 37 (1988) 443.

[3] M. Kato and K. Machida, Phys. Rev. B 37 (1988) 1510 ; C. Geibel et al, Z. Phys. B 86, 161 (1992) ; R. Caspary et al., Phys. Rev. Lett. 132146 (1993).

[4] D. W. Murphy et al., Phys. Rev. Lett. 58, 1888 (1987) ; J. W. Mills et al., Jr. Magn. \& Magn. Mat. 67 L 139 (1987).

[5] R. Nagarajan et al., Phys. Rev. Lett. 72, 274 (1994) ; R. J. Cava et al., Nature 367, 146 (1994) ; H. Eisaki et al., Phys. Rev. B 50, 647 (1994).

[6] K. Kitazawa et al., Jpn. Jr. Appl. Phys. 26, 339 (1987).

[7] Z. -X. Shen et al., Science 267, 343 (1995) ; H. Ding et al., Phys. Rev. Lett. 76 (9) 1533 (1996); S. LaRosa, I. Vobornik, F. Zwick, H. Berger, M. Grioni, G. Margaritondo, R. J. Kelley, M. Onellion ans A. Chubukov, Phys. Rev. B 56 R525 (1997).

[8] G. Shirane et. al. Phys. Rev. Lett 59, 1613 (1987); J. Transquada et. al. Phys.Rev. Lett. 60, 159 (1988).

[9] K. B. Lyons et. al. Phys. Rev. B 37, 2353 (1988); Phys. Rev. Lett. 60, 732 (1988).

[10] P. W. Anderson, Science 235, 1196 (1985).

[11] P. Monthoux and D. Pines, Phys. Rev. B 47, 6069 (1993) and references therein.

[12] J. R. Schrieffer, X. G. Wen, Phys. Rev. Lett. 60, 944 (1988) ; Phys. Rev. B 39, 11663 (1989).

[13] Haranath Ghosh and M. Sardar, Physica C 246, 335 (1995).

[14] J. Ma et al., Science 267,865 (1995) ; M. Ichioka, E. Enomoto, N. Hayashi and K. Machida, Phys. Rev. B 53 (5) 2233 (1996); Haranath Ghosh, Europhys. Lett. 43, 707 (1998); Haranath Ghosh, Phys. Rev. B 59, 3357 (1999).

[15] W. E. Pickett, Rev. Mod. Phys. 61, 433 (1989).

[16] C. M. Verma, S. Schmitt-Rink and E. Abrahams, Solid State Comm. 62, 681 (1987).

[17] Sudip Chakraborty et al., Science 261, 337 (1993).

[18] I. E. Dzyaloshinskii and V. M. Yakovenko, Zh. Eksp. Teor. Fiz., 94, 344 (1988).

[19] J. Ruvalds, C. T. Rieck, S. Tewar, J. Thoma and A. Virosztek , Phys. Rev. B 51, 3797 (1995).

[20] D. J. Scalapino, Phys. Rep. 250329 (1995).

[21] K. Machida, J. Phys. Soc. Jpn. 502195 (1981) ; S. N. Behera and S. Bhattacharya, Physica C 167, 112 (1990).

[22] S. N. Behera and S. G. Mishra, in "Theoretical and experimental aspects of valance Fluctuations and Heavy Fermions", Eds. L. C. Gupta and S. K. Mallik (Plenium Press, N Y) p-265 (1987) ; S. N. Behera and Haranath 
Ghosh, Z. Phys. B 95, 275 (1994).

[23] C. O'Donvan and J. P. Carbotte, Physica C 25287 (1995) ; Y. Ren et al., Phys. Rev. B 53, 2249 (1996)

FIG. 1. Amplitudes of the SDW and the $s$-wave SC gap evaluated at $5 \mathrm{~K}$, as a function of the hole concentration $(x)$.

FIG. 2. The SDW and extended $s$-wave gap amplitudes evaluated at $5 \mathrm{~K}$ is plotted as a function of the hole concentration $(x)$.

FIG. 3. Gap amplitudes of the SDW and the $d_{x^{2}-y^{2}}$ SC gap a $\mathrm{s}$ a function of hole concentration $(x)$.

FIG. 4. The SDW and SC gap in the mixed $(s+d)$ symmetry is plotted as a function of the hole concentration.

FIG. 5. Thermal variation of the isotropic $s$-wave SC gap and the SDW gap (in units of $t$ ) for various fixed hole concentrations $(x)$.

FIG. 6. Temperature variation of the isotropic $s$-wave SC gap and the SDW gap for $x=0.1$. The thermally instable region is worth noting.
; K. A. Musaelian et al., Phys. Rev. B 53, 3598 (1996).

[24] L. C. Gupta, Physica B 223 - 224, 56 (1996). 


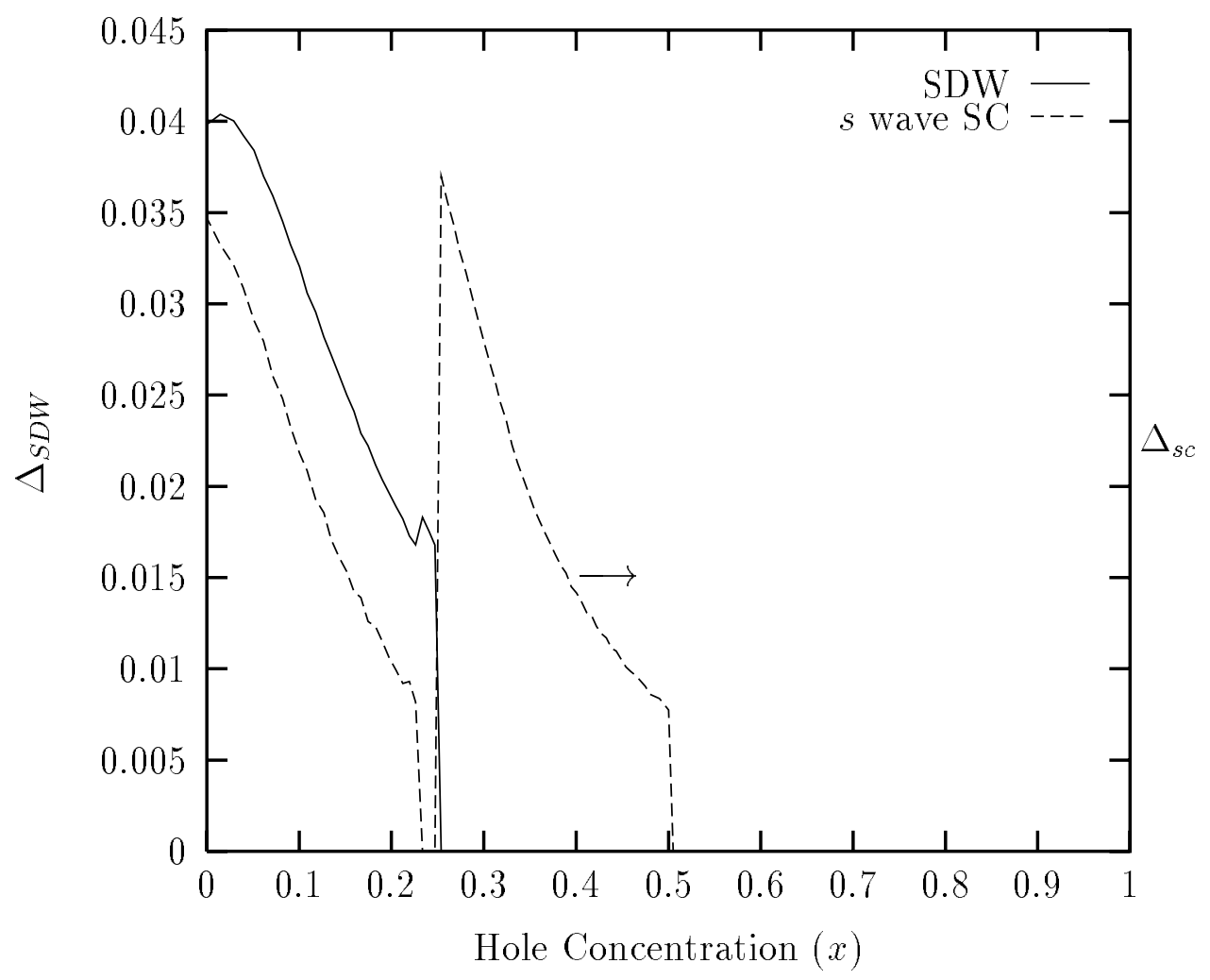




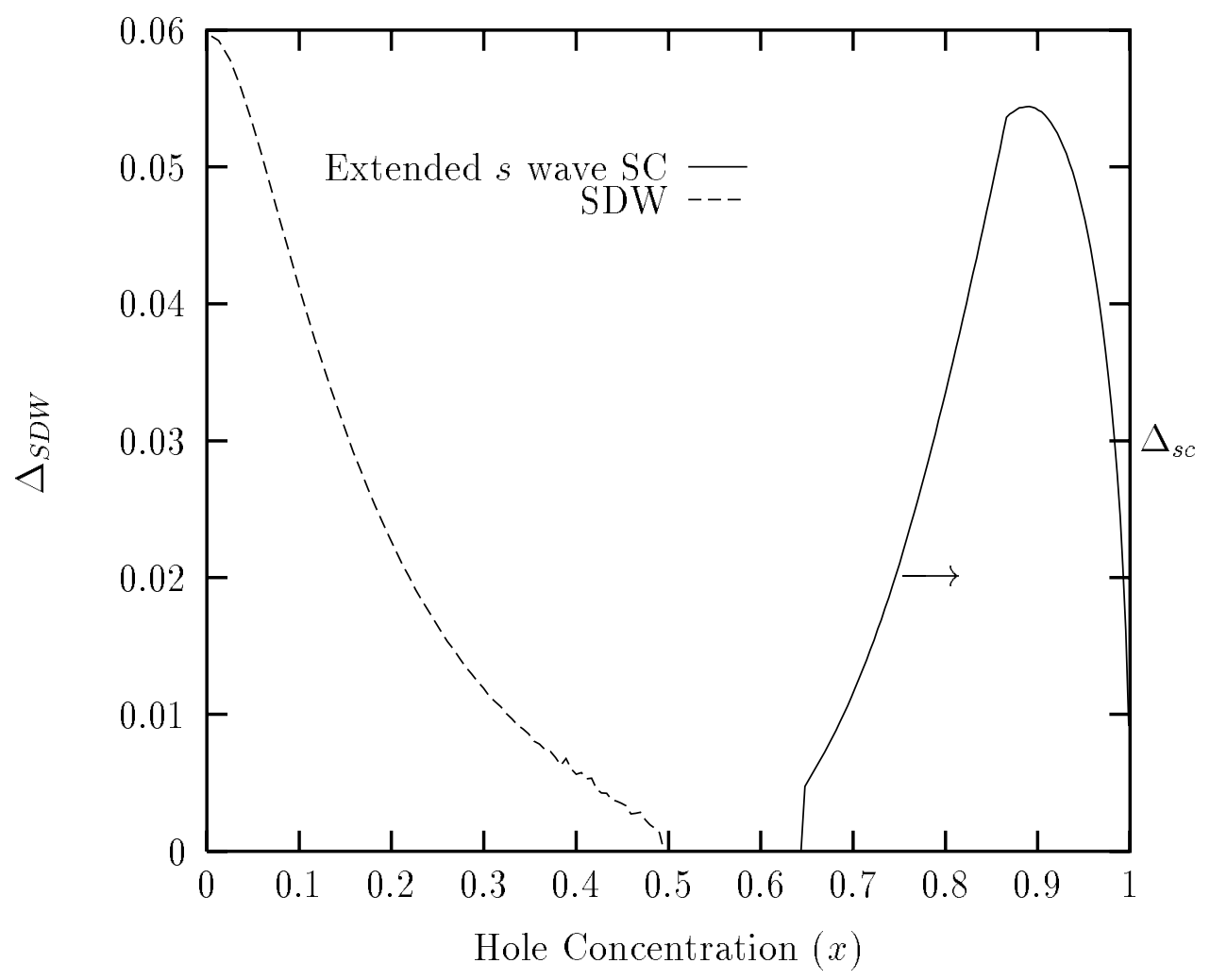




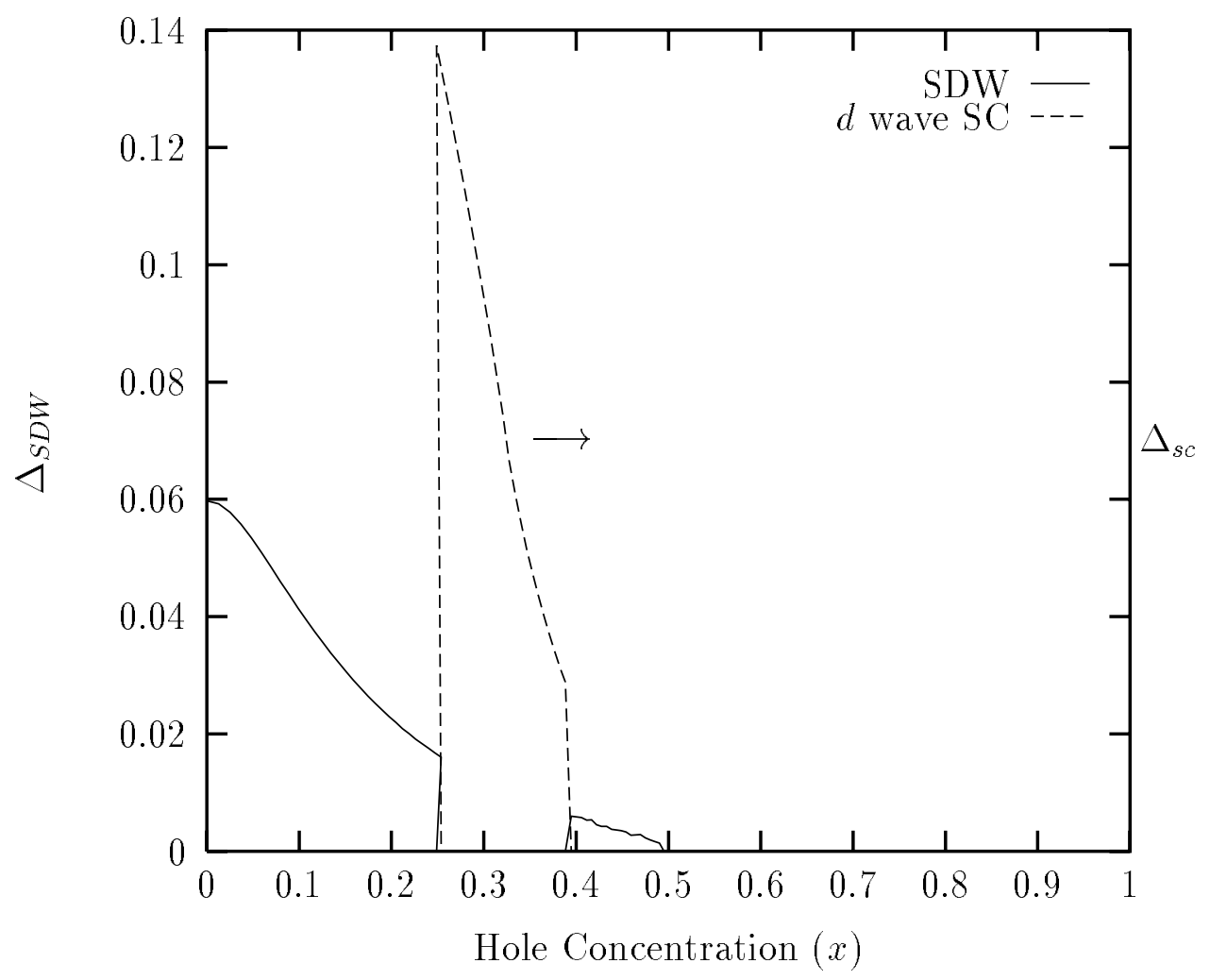




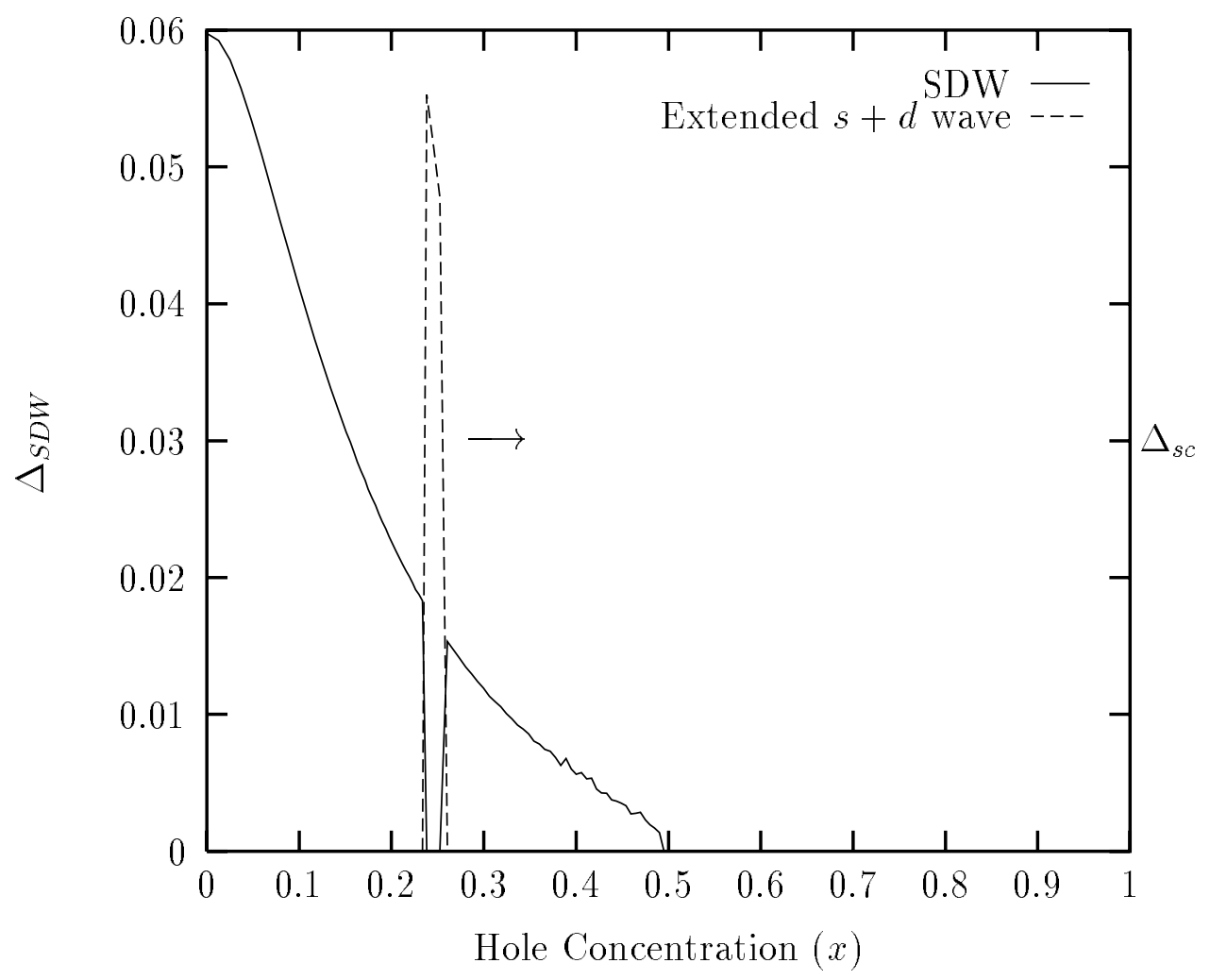




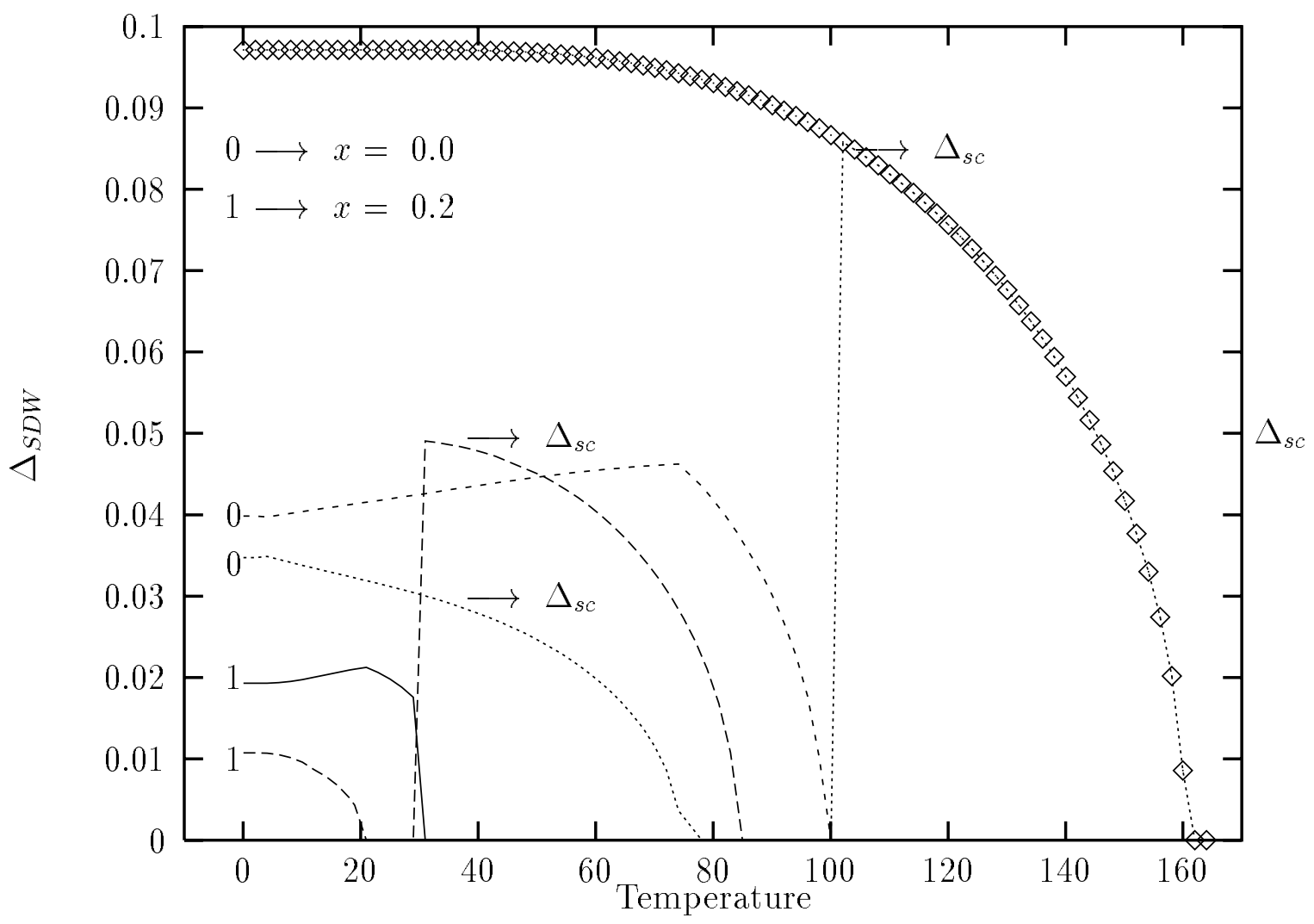




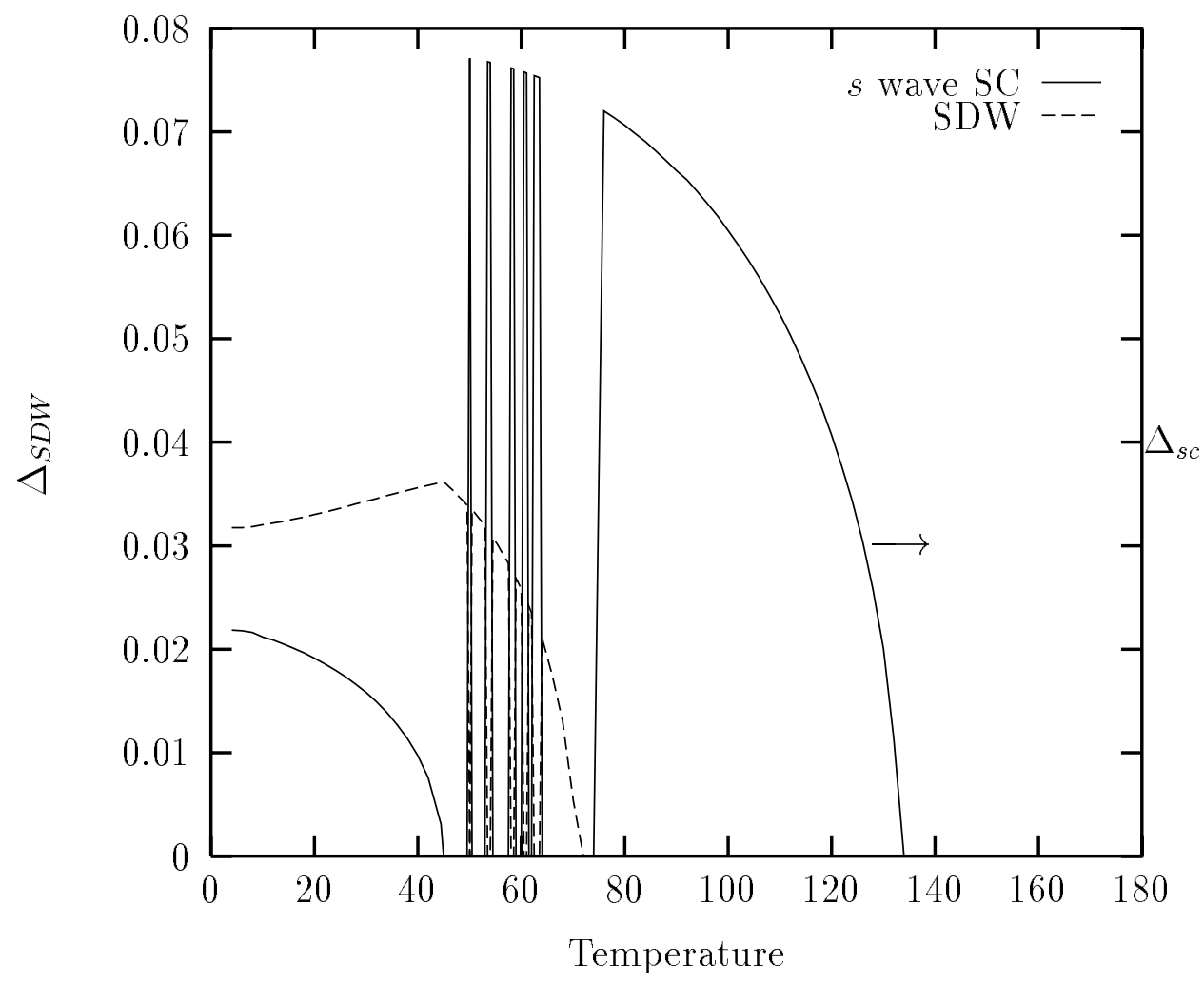

